\title{
Further evidence for a late locus of holistic word processing: Exploring vertex effect in the word composite task
}

\author{
Paulo Ventura ${ }^{1}$ (1) - João Delgado ${ }^{1}$ • José C. Guerreiro ${ }^{1} \cdot$ Francisco Cruz ${ }^{1} \cdot$ Vivienne Rosário $^{1}$. \\ António Farinha-Fernandes ${ }^{1} \cdot$ Miguel Domingues $^{1} \cdot$ Ana Margarida Sousa $^{1}$
}

Published online: 30 August 2020

(C) The Psychonomic Society, Inc. 2020

\begin{abstract}
Previous studies have shown a rather late and lexical level for holistic word processing. In the present study, we evaluated whether there are early effects in holistic processing of words, taking into consideration the role of lower-level visual processes that are critical in the hierarchy of visual word recognition: the extraction of viewpoint-invariant line junctions/vertices. We used contour-deleted words in two conditions: preservation of the vertices versus preservation of midsegments and an all-contour condition. We found evidence of a composite effect that was equivalent for all materials. Thus, we found no evidence of an early contribution of holistic processing to word recognition, and confirmed that holistic word processing is related to late lexical orthographic representations.
\end{abstract}

Keywords Visual word recognition · Psycholinguistics · Orthography

\section{Introduction}

Faces are homogeneous visual objects with which we all have extensive experience, but their individuation is crucial in our lives. Holistic processing (Richler, Palmeri, \& Gauthier, 2012), or the consideration of all parts together, is at the base of this individuation ability. Holistic processing has also been observed for naturally occurring objects, but only for experts in those objects (e.g., X-rays: Bilalic, Grottenthaler, Nagele, \& Lindig, 2014; chessboards: Bilalic, Langner, Ulrich, \& Grodd, 2011; fingerprints: Busey \& Vanderkolk, 2005; cars: Gauthier, Curran, Curby, \& Collins, 2003). Thus, it seems that holistic processing develops through extensive experience.

Words are also homogeneous visual objects that are made of a limited set of letters. And, indeed, evidence for holistic processing of words has been found in English words (Wong

João Delgado, José C. Guerreiro, Francisco Cruz, Vivienne Rosário, António Farinha-Fernandes, Miguel Domingues and Ana Margarida Sousa contributed equally to this work.

Paulo Ventura

paulo.ventura@gmail.com

1 Faculdade de Psicologia, Universidade de Lisboa, Alameda da Universidade, 1649-013 Lisbon, Portugal et al., 2011), Chinese characters (Wong et al., 2012), and Portuguese words (Ventura et al., 2017).

Both holistic face and holistic word processing have been studied using a task called the composite paradigm: for example, participants are asked to decide whether the left part of two words presented in sequence is the same while ignoring the right part. Any interference in performance from the irrelevant part on the relevant part indicates automatic and compulsory processing of all parts of the stimuli. In the complete version of this task (Richler \& Gauthier, 2014), there are four conditions, including "same" and "different" trials (the critical halves of the two stimuli being same or different) with irrelevant halves that are different or the same. One can thus define "congruent" and "incongruent" trials depending on the relation between the correct response for the target part and the same/different status of the task-irrelevant part (Richler \& Gauthier, 2014). The complete paradigm usually involves an aligned (parts are aligned) and a misaligned (parts are misaligned) condition. Holistic processing is inferred from a better performance on congruent than incongruent trials that is decreased in misaligned trials.

In the discussion above, we provided evidence for the idea that holistic processing style develops through extensive experience. However, Zhao et al. (2016) reported face-like composite effects for novel Gestalt-line patterns with salient Gestalt information (i.e., connectedness, closure, and 
continuity between parts) in the absence of expertise. Therefore, face-like holistic processing is not limited to faces and objects of expertise. These results are consistent with a dual-route account of holistic processing (Zhao et al., 2016), with a bottom-up (stimulus based) and top-down (experiencebased) route to holistic processing.

Curby and Moerel (2019) followed up on the Zhao et al. study by examining whether one can observe a trade-off in holistic processing indices for faces and Gestalt stimuli in a task designed to tap an overlap in early perceptual processing stages supporting Gestalt perception. Faces were processed less holistically when an aligned (processed holistically), compared with a misaligned (not processed holistically), Gestaltline pattern was overlaid, and Gestalt-line patterns were processed less holistically when an aligned (processed holistically), compared to a misaligned (not processed holistically), face was overlaid. This overlap in the holistic processing of faces and stimuli strong in Gestalt cues suggest an overlap at earlier, more perceptual, processing stages that could support a stimulus-based contribution to holistic processing.

There is also some evidence for a dual-route account of holistic processing of words with evidence for both an earlier and a late locus for the word-composite effect. First, some work points to a late, lexical effect. Ventura et al. (2017) specifically investigated whether the word-composite effect was affected by low-level visual aspects of stimuli, using words in three fonts: courier, notera, and aLtErNaTiNg-case courier. Courier is a typical printed font, but both notera and alternating-case words differ from typical, printed words in geometrical structure and perceptual difficulty, making visual word recognition more difficult. Ventura et al. (2017) showed that the composite effect was similar for the three types of words, suggesting an involvement at the level of abstract lexical representations. In a second study, Ventura et al. (2019), evaluated whether contextually induced congruency effects can occur within a single trial between words and artificial objects. These context effects were previously found by Richler, Bukach, and Gauthier (2009) with faces and Greebles. Ventura et al. (2019) used a different type of artificial objects, Ziggerins (Wong et al., 2009), and found no evidence that an aligned word (which is processed holistically) induces a stronger congruency effect on artificial objects than on aligned pseudowords (which are not processed holistically). Given the time one trial takes in this task (but in accordance with those used by Richler et al., 2009), holistic word processing may reflect rather late linguistic, lexical/ orthographic processes, which may not be abstract enough to allow an influence on other, nonlinguistic categories.

Secondly, there appears to be some evidence for an earlier locus of the word-composite effect. Indeed, Chen et al. (2013) showed in an event-related potential (ERP) study with Chinese characters that holistic processing of words has an earlier neurophysiological correlate (P1) than that (N170) commonly found for face holistic processing (e.g., Jacques \& Rossion, 2009), suggesting involvement of the early visual processes. Chen et al. (2016) found independence of presentation duration for Chinese characters in the composite task: variation in the exposure duration between 170 and $600 \mathrm{~ms}$ did not bring about significant changes in the holistic word effect.

Chen et al. (2013) and Chen et al. (2016) provided relatively indirect and non-linguistic evidence of an early locus on holistic word processing. In the present study, we evaluated more directly whether there is a true early effect in holistic processing of words, taking into consideration the role of lower-level, visual processes that are critical in the hierarchy of visual word recognition (Dehaene, Cohen, Sigman, \& Vinckier, 2005): the extraction of viewpoint-invariant line junctions/vertices.

Indeed, in word processing, we have not only an abstract level, but also lower-level visual processes that are common to visual recognition. Biederman investigated the role of invariant properties in human object recognition. Biederman (1987) used as base figures line drawings of objects and removed an equal amount of contour either at their vertices or at their midsegments. When vertices were deleted from the drawing of an object, object recognition was more impaired than when an equivalent proportion of contour was deleted from the midsegments, sparing the vertices. Thus, viewpoint-invariant vertex configurations play a crucial role in visual object recognition. The same vertex effect has been observed for words (Lanthier, Risko, Stolz, \& Besner, 2009; Szwed, Cohen, Qiao, \& Dehaene, 2009). The version with intact vertices and degraded mid-segments was recognized better than the version with intact mid-segments and degraded vertices. This vertex effect has been observed with very short presentations (50 ms; Lanthier et al., 2009; $100 \mathrm{~ms}$; Cohen et al., 2009). Thus, the effect probably occurs at an early level of visual word processing and its importance, as for objects, arises probably because vertices are relatively invariant properties that are common to most viewpoints. This is consistent with the proposition of a hierarchical arrangement of several levels of the visual system in which extraction of vertices precedes more abstract recognition of letter identities (Dehaene, Cohen, Sigman, \& Vinckier, 2005).

In the current study we evaluated whether an early mechanism of the hierarchical processing of visual words - the extraction of viewpoint-invariant line junctions/vertices, which most probably has its role before more abstract levels in the hierarchy of visual word recognition influence the wordcomposite effect. Holistic processing might intervene to bind together view-point-invariant line junctions, providing the input that activates abstract letter identities.

We thus used the vertex effect to evaluate whether we can find evidence of an involvement of early visual word features in holistic processing of words. We presented participants 
with an intact version and two degraded versions of the words, one in which part of the vertices were deleted but the midsegments were preserved, and one in which part of the midsegments were deleted but the vertices were preserved. The amount of contour removed in both versions was the same. If there is an early locus for the word-composite effect, the composite effect might be smaller/disrupted when vertices are removed. These results would suggest that vertices play an important role in word holistic processing. If the locus of the holistic word effect resides only in a late abstract and lexical locus, we expected no differences between the version with vertices deleted and the version with mid-segments deleted.

\section{Method}

\section{Participants}

Sixty-six participants were part of the Intact Contour group, 65 participants were part of the Vertex Preserved group, and 73 participants were part of the Mid-segment Preserved group. A further four participants in the Intact Contour group, seven participants in the Vertex Preserved group, and seven participants in the Mid-segment Preserved group were removed from the analyses due to excessive error rates $(>30 \%)$. According to G*Power (Version 3.1; Faul, Erdfelder, Buchner, \& Lang, 2009), a sample size of 52 would be required for each Feature Type group to detect a medium-sized effect (with $\eta p 2=.05$ ) at $\alpha=0.05$ with a power of 0.9 for a repeated-measures ANOVA with a two-level within-subject factor

Participants received a course credit.

This study's protocol adhered to the guidelines of the Declaration of Helsinki and the Portuguese deontological regulation for Psychology, and was approved by the Deontological Committee of Faculdade de Psicologia of Universidade de Lisboa. All participants provided written informed consent.

\section{Materials and procedure}

Stimuli were presented on a 17 -in. CRT monitor, and E-Prime 2.0 was used to control stimulus presentation and responsetime recording.

The task was identical to that in Ventura et al.'s (2017) Experiment 1 (cf. Fig. 1 in that article), but the events on a trial were presented at a much faster rate. Two words were shown sequentially (the study and test words were presented for $250 \mathrm{~ms}$ and separated by a 500-ms mask). Response times (RTs) were collected from the onset of the test word, and participants had to judge if the target parts (always the left halves) of the two words were identical or not by pressing the "1" or "2" key (with a green or red label) as quickly and

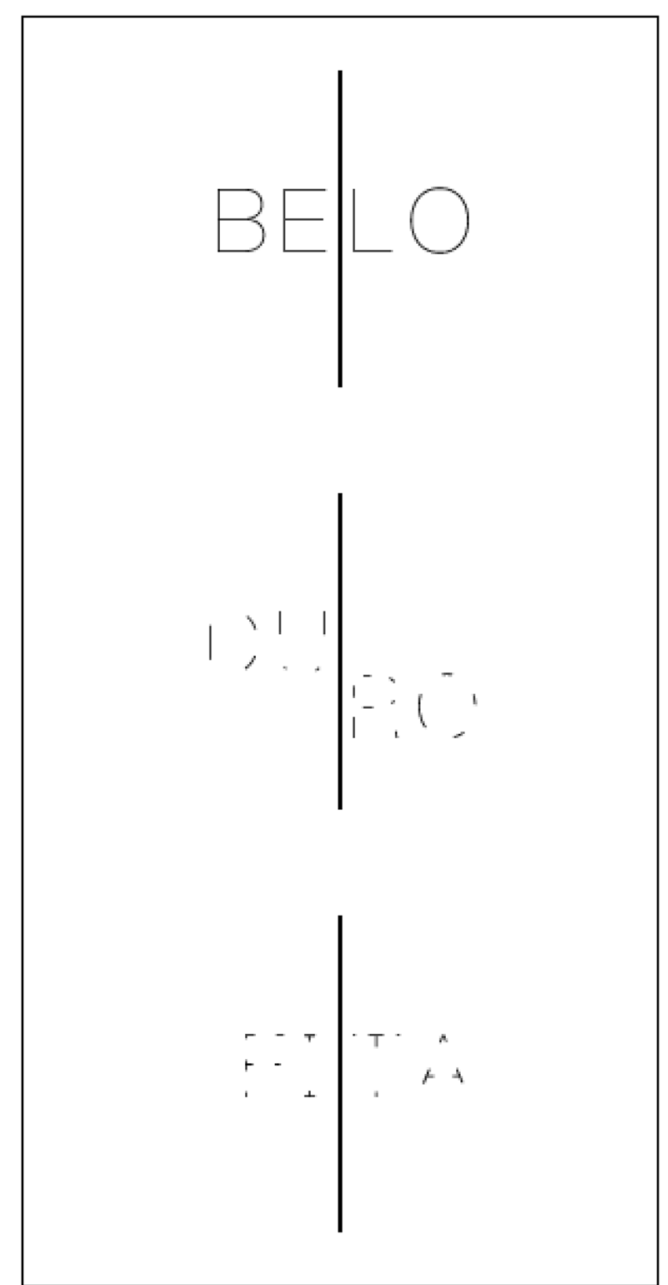

Fig. 1 From top to bottom, examples of Intact Contour stimuli (aligned), Mid-segment preserved (misaligned), and Vertex preserved (aligned) materials

accurately as possible, while ignoring the other, irrelevant part. After $2.5 \mathrm{~s}$ or upon response, the stimuli disappeared and a blank screen appeared for $500 \mathrm{~ms}$ before the beginning of the next trial. The task adopted the complete version of the composite paradigm (Richler \& Gauthier, 2014). In this version, one can define four types of trial: same-congruent, sameincongruent, different-congruent, and different incongruent. In half of the trials the target and irrelevant parts are aligned with each other and in the other half they are misaligned. Holistic processing is typically inferred from an interaction between alignment and congruency (e.g., Richler, Mack, et al., 2011): better performance in congruent than incongruent trials especially when the parts are aligned as opposed to misaligned.

The stimuli were 20 sets of four-letter (consonantvowel.consonant-vowel) CV.CV Portuguese words. The same 18 different uppercase letters were used to construct the words. The letters were predominantly non-curvy (A, B, D, E, F, G, I, J, L, M, N, P, R, T , U, V, X) with the exception 
of "O," which had to be used in some words due to constraints of the material. For all stimuli, we used a sans serif font with thin lines (Helvetic Ultra Light, 42 point); serif was added to the letter "I."

These stimuli could be either intact or degraded by the removal of fragments. Two modes of degradation were used: Compared with the intact stimulus (Intact Contour; Fig. 1, top), in the vertex-deleted variant, the mid-segments were preserved but the vertices were deleted (Mid-segment; Fig. 1, middle), whereas in the mid-segment-deleted variant, the vertices were preserved but the mid-segments were deleted (Vertex; Fig. 1, bottom). In both cases, an equal proportion of contour was deleted: $65 \%$ of the original. This choice related to the fact that Szwed et al. (2009) were only able to elicit a vertex effect with more degraded stimuli (65\% vs. $45 \%)$.

Each word was divided into the left and right halves by a vertical line. Within each set, the left and right halves of each word were interchanged to create the four words resulting from the orthogonal manipulation of response (same; different) and congruency (congruent; incongruent): for example, vida, muro, viro, muda. Each word appeared both as study and test stimuli. Thus, the same distractor parts were used in different conditions (congruent and incongruent). Participants performed eight blocks of trials (four aligned; four misaligned; block- and trial-order randomized), each with 80 trials. Before the experimental trials, participants were first presented with four examples on paper, for which they received feedback on the correct response. Next, they performed 16 computerized practice trials with different stimuli.

\section{Results}

We performed an overall ANOVA for ACC and another for RTs, including the repeated measures of Alignment (aligned, misaligned), Congruency (congruent, incongruent), Response Type (different, same) and the between-subjects factor of Feature Type (Intact Contour, Midsegment Preserved, and Vertex Preserved).

In the ANOVA run for accuracy, there was a significant effect of Congruency, $F(1,208)=49.65, p<.0001$, partial $\eta^{2}$ $=.19$. There was also an effect of Response Type, $F(1,208)=$ $41.72, p<.0001$, partial $\eta^{2}=.17$, with "same" responses (.95) being less accurate than "different responses (.97). There was also an interaction of Alignment, Response Type, and Material, $F(1,208)=3.15, p=.045$, partial $\eta^{2}=.03$, Although this interaction explains only a small portion of the variance, we tried to interpret it. In the All Contour condition, there is no difference between "different" and "same" accuracy either in the aligned or the misaligned conditions. In the Mid-segment Preserved condition, there is a difference between "different" (more accurate) and "same" responses. This difference is higher in the aligned compared to the misaligned condition. The same pattern was found in the Vertex Preserved condition This difference is higher in the aligned compared with the misaligned condition. Four-way interaction was close to $1, F=.94$. No other effects were significant (Tables 1 and 2).

In the ANOVA run for RTs, we found an effect of Feature Type, $F(2,208)=9.6, p<.0001$, partial $\eta^{2}=.08$. Bonferronicorrected tests showed All Contour (633.04) to have faster performance than both Vertex preserved (702.74) and Midsegment preserved (732.42), while performance in Vertex preserved was equivalent to Mid-segment preserved. The effects of Alignment, $F(1,208)=5.52, p=.02$, partial $\eta^{2}=.03$, and Congruency, $F(1,208)=40.14, p<.0001$, partial $\eta^{2}=.16$, were significant. The interaction of Alignment and Congruency was significant, $F(1,208)=11.98, p<.001$, partial $\eta^{2}=.05$. This interaction is known as the composite effect. Holistic processing is inferred from a better performance on congruent than incongruent trials that is reduced in misaligned trials (15 ms in aligned vs. $6 \mathrm{~ms}$ in misaligned).

As regards Response type, there was a significant effect, $F(1,208)=66.45, p<.0001$, partial $\eta^{2}=.24$, with "same" responses faster (677.97) versus "different" responses (700.83). Response Type interacted with Congruency, $F(1$, 208) $=11.13, p<.001$, partial $\eta^{2}=.05$, with a stronger congruency effect for "different" responses $(27 \mathrm{~ms})$ than for "same" responses (18 ms). There was also a triple interaction between Alignment, Congruency, and Response Type, $F(1$, 208) $=15.21, p<.0001$, partial $\eta^{2}=.07$, with a stronger interaction of Alignment and Congruency for "same" responses than for "different" responses.

The four-way interaction was $<1$. No other effects were significant.

Thus, we found evidence of a composite effect (Alignment $\times$ Congruency) that is equivalent for the three Feature Types. To further evaluate this conclusion, we used a planned contrast with one degree of freedom comparing the composite

Table 1 Accuracy for the main conditions of the study. Standard error of the mean is given in parentheses

\begin{tabular}{lcll}
$\begin{array}{l}\text { Intact contour } \\
\text { Aligned }\end{array}$ & & Misaligned & \\
Congruent & Incongruent & Congruent & Incongruent \\
$.97(.004)$ & $.96(.005)$ & $.97(.004)$ & .96 \\
Vertex preserved & & & \\
Aligned & & Misaligned & \\
Congruent & Incongruent & Congruent & Incongruent \\
$.97(.004)$ & $.96(.005)$ & $.97(.004)$ & $.96(.005)$ \\
Mid-segment preserved & & \\
Aligned & & Misaligned & \\
Congruent & Incongruent & Congruent & Incongruent \\
$.97(.003)$ & $.96(.004)$ & $.97(.004)$ & $.96(.004)$ \\
\hline
\end{tabular}


effect for All Contour versus both Vertex preserved and Midsegment preserved, which was not significant. Numerically, the size of the composite effect is very similar across the three Feature Types. Considering aligned trials, the congruency effect is $15 \mathrm{~ms}$ for All Contour, $17 \mathrm{~ms}$ for Vertex preserved, and $12 \mathrm{~ms}$ for Mid-segment preserved. Any interpretation of the results in terms of easiness of perceptual processing (participants of the easier task - All Contour - might have done more processing of the irrelevant information) are incompatible with the fact that the Alignment effect and Alignment $\times$ Congruency interaction is very similar for all Feature Types.

We further adopted an individual differences approach and regressed out the congruency effect (difference in performance between congruent and incongruent trials) for the misaligned trials from the congruency effect for the aligned trials, and obtained the residuals of that regression, which may constitute a purer measure of the composite effect. Comparing the residuals across the three materials revealed no differences, $\mathrm{F}<1$ (Fig. 2).

\section{Discussion}

The aim of the present work was to evaluate whether an early pathway may contribute to holistic word processing, in the same fashion as that proposed for faces; indeed, Zhao et al. (2016) proposed a dual-route account including a stimulus-based and an experiencedbased route. We used contour-deleted words in two conditions: preservation of the vertices versus preservation of mid-segments. Vertices of words are extracted early before the computation of abstract letter identities (Dehaene, Cohen, Sigman, \& Vinckier, 2005), and its importance, as for objects, arises probably because vertices are relatively invariant properties that are common to most viewpoints. We used the composite paradigm with these two materials and a condition in which all of the contour was preserved.

We found no evidence suggestive of differential composite effects for the three materials. Viewpoint-invariant vertex configurations no doubt play a crucial role in visual word recognition (Lanthier, Risko, Stolz, \& Besner, 2009; Szwed, Cohen, Qiao, \& Dehaene, 2009), but they do not seem important for holistic processing, which entails later, lexical stages.

It would nevertheless be interesting to run a study similar to Curby and Moerel (2019) evaluating whether other types of early information, like salient Gestalt information (i.e., connectedness, closure, and continuity between parts), interferes with holistic processing of (cursive words)

When considered together with previous findings (Ventura et al., 2017, 2019), these data suggest that holistic processing of words is related to abstract, lexical factors that can support holistic word perception. These results are also encompassed
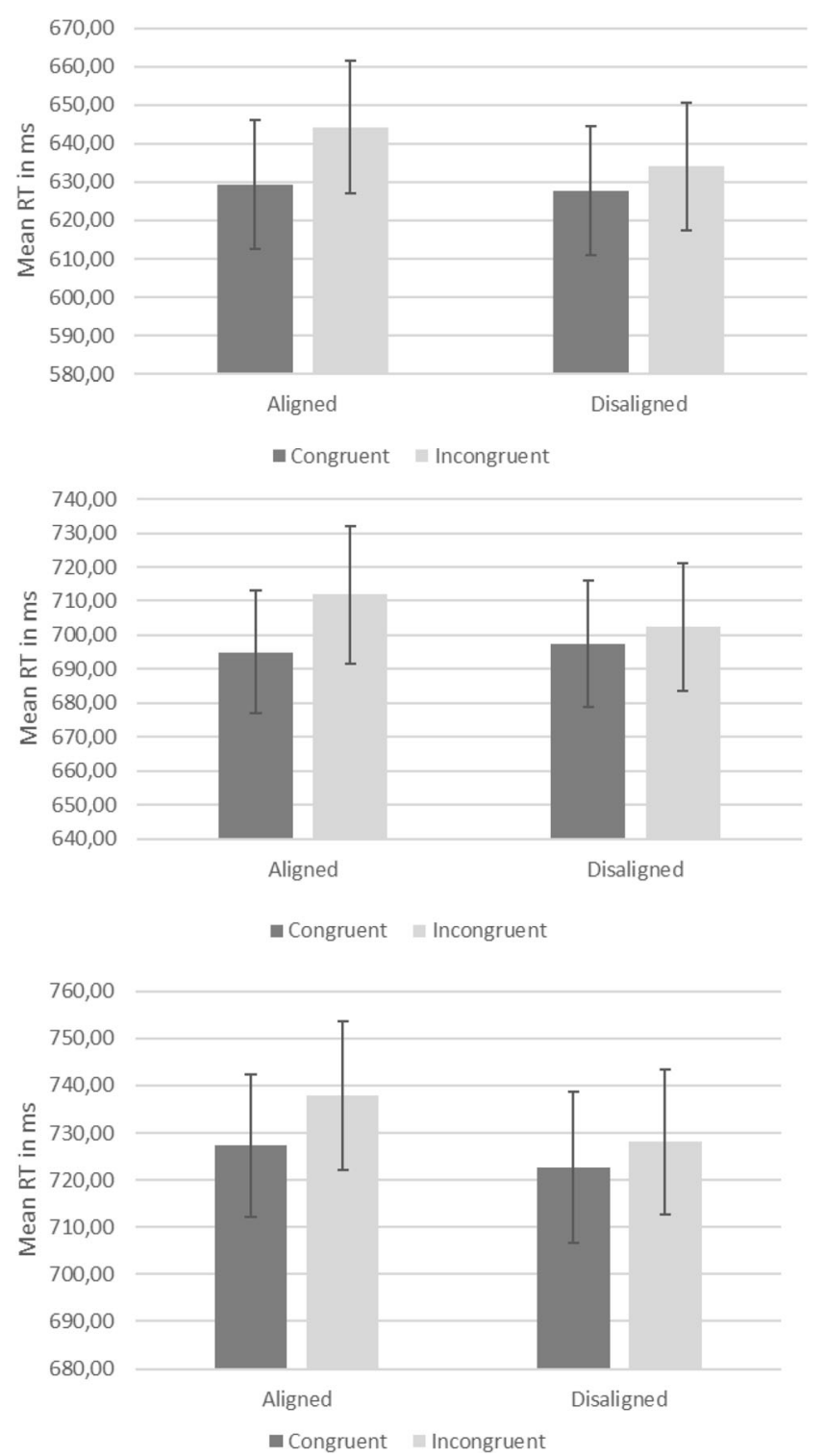

Fig. 2 Illustration of the interaction of Alignment $\times$ Congruency, separately for Intact Contour stimuli, Vertex preserved, and Midsegment preserved materials

by recent literature showing the relevance of holistic word representations in reading. Indeed the lateral anterior region of the VWFA is sensitive to lexical properties and underpins holistic word representations, the most posterior region of VWFA is sensitive to sublexical orthographic representations (Bouhali et al., 2019; Lerma-Usabiaga et al., 2018).

One should not forget that in visual word recognition it has long been shown that letter identities are not bypassed and word holistic processing is not just about supra letter features (e.g., Paap, Newsome, \& Noel, 1984). The word-composite effect is in no way related to these ideas. The composite effect shows that all parts of a visual word are fully processed even if the task requires a decision on a part only. Holistic processing reflects obligatory encoding of/attending to all object parts, 
which in turn are also encoded and represented independently (Richler \& Gauthier, 2014).

In sum, our results confirm that holistic processing of words involves abstract, orthographic, and lexical representations.

Acknowledgements The work presented in this article was supported by the Research Center for Psychological Science (CICPSI) at Universidade de Lisboa.

Table 2 Words used in the composite task

\begin{tabular}{|c|c|c|c|}
\hline 舄Bola (ball) & 舄Mude (change it) & 舄Bode (goat) & 舄Mula (mule) \\
\hline 舄Faro (nose) & 舄Pule (jump) & 舄Fale (speak) & 舄Puro (pure) \\
\hline 舄Fuga (escape) & 舄Tome (take) & 舄Fume (smoke) & 舄Toga (gown) \\
\hline 舄Lixo (trash) & 舄Puma (puma) & 舄Lima (lime) & 舄Puxo (pull) \\
\hline 舄Mito (myth) & 舄Gare (dock) & 舄Mire (aim) & 舄Gato (cat) \\
\hline 舄Pura (pure) & 舄Belo (beautiful) & 䳔Pulo (jump) & 舄Bera (bera) \\
\hline 舄Tido (had) & 舄Lave (wash) & 舄Tive (I had) & 舄Lado (side) \\
\hline 舄Tiro (shot) & 舄Duna (dune) & 舄Tina (tub) & 舄Dure (last) \\
\hline 舄Tive (I had) & 舄Mora (lives) & 舄Tira (strip) & 舄Move (move) \\
\hline 舄Vida (life) & 舄Muro (wall) & 舄Viro (turn) & 舄Muda (changes) \\
\hline 舄Vila (village) & 舄Fure (drill) & 舄Vire (turn) & 舄Fula (angry) \\
\hline 舄Foge (run away) & 舄Mura (wall) & 舄For a (out) & 舄Muge (moan) \\
\hline 舄Lido (read) & 舄Jura (swear) & 舄Lira (lira) & 舄Judo (judo) \\
\hline 舄Figo (fig) & 舄Juta (jute) & 舄Fita (ribbon) & 鳥Jugo (yoke) \\
\hline 舄Fuma (smoke) & 舄Rijo (tough) & 舄Fujo (run away) & 舄Rima (rime) \\
\hline 舄Lima (lime) & 舄Rude (rude) & 舄Lide (deal) & 舄Rumo (course) \\
\hline 舄Luto (mourning) & 舄Ripa (clapboard) & 舄Lupa (magnifying glass) & 舄Rito (rite) \\
\hline 舄Mero (mere) & 舄Fulo (angry) & 舄Melo (melon) & 舄Fura (punctures) \\
\hline 舄Neta (granddaughter) & 舄Fila (row) & 舄Nega (deny) & 舄Fito (regard) \\
\hline 舄Vime (wicker) & 舄Ruga (wrinkle) & 舄Viga (beam) & 䳔Rume (course) \\
\hline
\end{tabular}

\section{References}

Biederman, I. (1987). Recognition-by-components: A theory of human image understanding. Psychological Review, 94, 115-147. doi: https://doi.org/10.1037/0033-295X.94.2.115

Bilalic, M., Grottenthaler, T., Nagele, T., and Lindig, T. (2014). The Faces in Radiological Images: Fusiform Face Area Supports Radiological Expertise. Cerebral Cortex, 26, 1004-1014. doi: https://doi.org/10.1093/cercor/bhu272

Bilalic, M., Langner, R., Ulrich, R., and Grodd, W. (2011). Many faces of expertise: fusiform face area in chess experts and novices. Journal of Neuroscience, 31, 10206-10214. doi:https://doi.org/10.1523/ jneurosci.5727-10.2011

Bouhali, F., Bézagu, Z., Dehaene, S., \& Cohen, L. (2019). A mesial-tolateral dissociation for orthographic processing in the visual cortex. Proceedings of the National Academy of Sciences, 201904184. doi: https://doi.org/10.1073/pnas.1904184116

Busey, T. A., and Vanderkolk, J. R. (2005). Behavioral and electrophysiological evidence for configural processing in fingerprint experts.
We would like to thank two anonymous reviewers for very helpful comments and suggestions.

Accessibility statement Research data and statistical data files are available at: DOI 10.17605/OSF.IO/DWSY6

\section{Appendix}


Faul, F., Erdfelder, E., Buchner, A., \& Lang, A. (2009). Statistical power analyses using $\mathrm{G}^{*}$ Power 3.1: Tests for correlation and regression analyses. Behavior Research Methods, 41, 1149-1160. https://doi. org/10.3758/brm.41.4.1149

Gauthier, I., Curran, T., Curby, K. M., and Collins, D. (2003). Perceptual interference supports a non-modular account of face processing. Nature Neuroscience, 6, 428-432. doi:https://doi.org/10.1038/ nn1029

Jacques, C., Rossion, B. (2009). The initial representation of individual faces in the right occipito-temporal cortex is holistic: Electrophysiological evidence from the composite face illusion. Journal of Vision, 9, 8, 1-16. https://doi.org/10.1167/9.6.8

Lanthier, S. N., Risko, E. F., Stolz, J. A., \& Besner, D. (2009). Not all visual features are created equal: Early processing in letter and word recognition. Psychonomic Bulletin \& Review, 16, 67-73. https://doi. org/10.3758/PBR.16.1.67

Lerma-Usabiaga, G., Carreiras, M., \& Paz-Alonso, P. M. (2018). Converging evidence for functional and structural segregation within the left ventral occipitotemporal cortex in reading. Proceedings of the National Academy of Sciences, 115(42), E9981-E9990. doi: https://doi.org/10.1073/pnas.1803003115

Paap, K. R., Newsome, S. L., \& Noel, R. W. (1984). Word shape's in poor shape for the race to the lexicon. Journal of Experimental Psychology: Human Perception and Performance, 10(3), 413-428. doi:https://doi.org/10.1037//0096-1523.10.3.413

Richler, J. J., Bukach, C. M., \& Gauthier, I. (2009). Context influences holistic processing of nonface objects in the composite task. Attention, Perception, \& Psychophysics, 71, 530-540. https://doi. org/10.3758/APP.71.3.530

Richler, J. J., \& Gauthier, I. (2014). A meta-analysis and review of holistic face processing. Psychological Bulletin, 140, 1281-1302. doi: https://doi.org/10.1037/a0037004

Richler, J. J., Mack, M. L., Palmeri, T. J., \& Gauthier, I. (2011). Inverted faces are (eventually) processed holistically. Vision Research, 51, 333-42. doi: https://doi.org/10.1016/j.visres.
Richler, J.J., Palmeri T. J., and Gauthier, I. (2012). Meanings, mechanisms, and measures of holistic processing. Frontiers in Psychology, 3. doi: https://doi.org/10.3389/fpsyg.2012.00553

Szwed, M., Cohen, L., Qiao, E., \& Dehaene, S. (2009). The role of invariant line junctions in object and visual word recognition. Vision Research, 49, 718-725. doi: https://doi.org/10.1016/j.visres. 2009.01.003

Ventura, P., Fernandes, T., Leite, I., Almeida, V. B., Casqueiro, I., \& Wong, A.C-N. (2017). The word-composite effect depends on abstract lexical representations but not surface features like case and font. Frontiers in Psychology, 8, 1036. doi: https://doi.org/10.3389/ fpsyg.2017.01036

Ventura, P., Pereira, A., Xufre, E. et al. Holistic word context does not influence holistic processing of artificial objects in an interleaved composite task. Attention Perception Psychophysics, 81, 17671780 (2019). https://doi.org/10.3758/s13414-019-01812-6

Wong, A. C.-N., Palmeri, T. J., \& Gauthier, I. (2009). Conditions for face like expertise with objects: becoming a Ziggerin expert-but which type? Psychological Science, 20(9), 1108-17. https://doi.org/10. 1111/j.1467-9280.2009.02430.x

Wong, A. C.-N., Bukach, C. M., Hsiao, J., Greenspon, E., Ahern, E., Duan, Y., \& Lui, K. F. H. (2012). Holistic processing as a hallmark of perceptual expertise for nonface categories including Chinese characters. Journal of Vision, 12. doi:https://doi.org/10.1167/12. 13.7

Wong, A. C.-N., Bukach, C. M., Yuen, C., Yang, L., Leung, S., \& Greenspon, E. (2011). Holistic Processing of Words Modulated by Reading Experience. PLoS One, 6, e20753. doi:https://doi.org/10. 1371/journal.pone.0020753

Zhao, M., Bulthoff, H. H., \& Bulthoff, I. (2016). Beyond faces and expertise: Facelike holistic processing of nonface objects in the absence of expertise. Psychological Science, 27(2), 213-222. doi: https://doi.org/10.1177/0956797615617779

Publisher's note Springer Nature remains neutral with regard to jurisdictional claims in published maps and institutional affiliations. 